\title{
Research on the Application of Feedback Mechanism in Product Interaction Design
}

\author{
Jiawei Song ${ }^{1, a}$ \\ ${ }^{1}$ Lanzhou Jiaotong University, Lanzhou, Gansu, China, 730070
}

\begin{abstract}
Keywords: Application, Feedback Mechanism, Product Interaction Design
\end{abstract}
\begin{abstract}
This paper first describes the application of feedback mechanism in product interaction design, analyzes the relationship between feedback behavior and its relationship with feedback behavior from the theoretical level. Finally, it analyzes the application mechanism of feedback behavior in product interaction design to achieve the product availability goals and improve the user experience.
\end{abstract}

\section{Introduction}

User-centered design has become the mainstream design trend, and ultimately the hope that the user's "usability" and "user experience" goals, Norman has put forward the "common position" concept to illustrate the lack of machine and human conversation, so that users often encounter complex product system to bring people's resistance, when users suffer from the product of the ever-changing problems, often become overwhelmed, difficult to deal with, which is mainly the designer failed to achieve an effective man-made two-way communication caused.

Successful interactive products should have a good ability to communicate with users and users need to encounter difficulties in the use of its correct guidance and eventually out of the woods. In the user want to get product feedback at the same time, people also hope that the product can take the initiative to send a signal of communication, feed forward is one of such a signal, feed forward the effective application allows users to more easily use the product, the occurrence of the problem has a certain ability to recover, so feed forward and feedback behavior, as in the interactive product design is indispensable.

\section{The Overview of Feedback Mechanism}

Feedback is an indication of the action that has occurred or is a response, and the feedback in the product is a collection of all feedback. When the product function is relatively simple, it can be embodied as a form of feedback, when the function is relatively complex, it can also be a combination of various forms of feedback. A good product must have a good feedback mechanism. It is the user behavior of the guidelines, that is, the user behavior design. When the user in the product operation, the product in a timely manner to the user's operation to make the appropriate feedback, so that users know the current state of the product. For the correct operation of the user, the product should make positive feedback to encourage the user to continue to operate; for the user wrong operation, the product in a friendly form to the user pointed out the error, and proposed to correct the wrong way.

\section{The Overview of Product Interaction Design}

The interactive design was presented as one of the founders of IDEO Bill Moggridge at a 1984 design meeting. The design of the interaction is largely due to the continuous application of the technology in the product and especially the microelectronics technology makes the computer chip in people's daily necessities widely used. The compactness of the product structure and the diversification of the function make the user's operation more complicated. When the human intelligence encounters the resistance of the complex system rules that change with the change of the problem, American scholar Cooper calls it "cognitive friction" [2], in order to solve this problem, 
interactive design came into being.

When the interactive design is presented, the content involved is quite extensive and is mainly used in the field of software design. Until recently, the interactive design has gradually penetrated from the field of computer science to the field of product design and development, and combined with product design theory Become a systematic approach. Using a systematic approach to thinking, the product interaction design system can be seen as a system of five basic elements (referred to as PACT-P) consisting of people, behavior, product use scenarios, and technologies that are fused in the product and the finished product.

Product interaction design to achieve the goal is to be based on user needs, to develop a product with availability, and ultimately achieve the goal of user experience. Popular said that the product first to "useful", and then to "easy to use", and finally to make users "want to use." It is clear that the requirements of the product from the material to the emotional level, so the impact of various factors in the system after a comprehensive consideration in the product interaction design feedback mechanism of the application, not only to meet the convenience function, but also reasonable, that is, through feedback to the user with the emotional two-way communication.

\section{The Feedback Mechanism Analysis of Product Interaction Design}

Feedback is a concept corresponding to the feed-forward, which is an indication of the action that has occurred or is a response [3], and the feedback mechanism in the product is a collection of all the feedback. When the product function is relatively simple, it can be embodied as a form of feedback, when the function is relatively complex, it can also be a combination of various forms of feedback. In the process of user interaction with the product, the product for each user's behavior should have a corresponding information feedback, said the user behavior of the recognition or denial, so that users get the results of the operation of the information. If the product cannot provide any feedback, then the user cannot determine the correctness of their operating behavior and the current product in what state, it is clear that such an interactive act is doomed to failure and this product is also unreasonable in the design. Therefore, in the product interaction design system, the feedback mechanism is an indispensable basic element and it is closely linked with the user behavior and together constitutes the core content of behavior design.

Based on the correctness of user actions, feedback can be divided into negative feedback and positive feedback. When the user performs the wrong operation behavior, the product can give the corresponding negative information feedback; when the user performs the correct operation behavior, the product can give the corresponding positive information feedback.

The Negative Feedback. Negative feedback is always a problem when the user behavior occurs, so naturally bear the role of the alarm. There is no doubt that people always do not want the product to convey negative feedback to themselves, because for the purpose of the alarm, the negative feedback is always in front of the user to show harsh, eye-catching, disturbing, noisy and even unpleasant form. For computer users, the most chilling and fear is accompanied by a low and short bang, the screen suddenly a dialog box, the above shows a bright red " $x$ " that the system has just occurred in the operation of the action. This allows users to instantly hate the high-tech troubles, while sighing why they make such a stupid action. If such negative feedback in every user is always appear, is bound to affect the user's emotional experience, interactive designers should reflect on this issue. In fact, this negative feedback in the computer is only a microcosm, in many high-tech products, filled with such behavior. For the user's emotional experience, in the product interaction design, in order to create user-centered human products, always do not want to embed negative feedback into the product.

However, in the design practice, the designer cannot ignore the negative feedback mechanism in the product interaction design system exists meaning. Negative feedback can play an irreplaceable role when warning users should not do the behavior or if the behavior can lead to undesirable consequences. For example, today's general use of alcohol on the car measuring instrument, once the driver's alcohol content is higher than the standard, it will issue a warning sound or the driver can not start the car and other negative feedback. Again, the gas-sensitive devices in the home, 
when the indoor gas content will exceed the excitement of the irritating taste, to cause the owner's vigilance. Negative feedback is more common for certain special populations or for specific situations.

Positive Feedback. If people are learning a dance, in a certain action on the error, the coach was shouting and severely criticized it, it is clear that this will not have any good results. In fact, in most cases, users are doing what they want to do, and accidental misuse will not cause serious consequences, so more negative feedback mechanism is not as good as the positive feedback mechanism is welcome. Any user who knows their own failure will feel frustrated, and after the success of the information will be confident and the negative feedback is so chilling. In the process of product interaction design, in order to achieve the ultimate goal of the user experience, we must try to inject emotional factors in the product, and ultimately embodies the joy of the product, so as to meet the user's emotional needs. In order to achieve this kind of emotional interaction between people and products, the use of positive feedback is essential. According to the product for the user behavior feedback information generated by the form of positive feedback can be divided into positive auditory feedback, positive visual feedback, positive olfactory feedback, position tracking and resistance feedback (for negative feedback, there are also auditory feedback, visual feedback and olfactory feedback, etc., only for a detailed analysis of positive feedback).

Positive Auditory Feedback. Well-known American scholar Cooper once said that almost every object and system outside the software world is to provide sound to express success, rather than failure, which fully shows that positive auditory feedback has a natural and pleasant. Close the door when you hear the tick that the door locked, and no sound that is not insurance; bath in the bathroom, open the fan, hear buzzing sound, said the fan in operation, no doubt, this proof Positive auditory feedback exists itself as a natural problem indicator. The higher level is that the product will provide the user with the music, the beautiful melody as the auditory information feedback, not only make the user feel the confidence of success, but also can experience the product to the user's emotions.

Positive Visual Feedback. Visual information is often reflected as bright light or not the light, the display shows the text or image information, color changes and the product's own action patterns and so on. Positive visual feedback in the daily life of the use of more frequently, press the computer monitor switch, the screen panel lights will be displayed as green; open the indoor fluorescent lamp, the switch will show around the weak yellow light; on the ATM for each operation behavior, the display will give the appropriate text or image feedback, on the contrary, then the description of the occurrence of misuse. At the same time, positive visual feedback can also be embodied as a beautiful animation demonstration and product form of performance, to give users the spirit of the enjoyment.

Resistance Feedback. To some extent, resistance feedback is more natural and subconsciously, it is natural in some products, it can be through the artificial design of such feedback mechanism into the product. Users in the operation through their own touch, feel the resistance changes in order to get feedback. Close the door when the power and the operation of the keys and so on the use of resistance feedback.

\section{The Application of Feedback Mechanism in Product Interaction Design}

Impact of Target Population. A systematic approach can help designers to clearly find revolutionary opportunities, and can also be an effective assessment of these ideas. When the user is positioned as a 7-person character and a targeted system design, the design team can roughly position the user as a target group based on the product interaction design system, based on the commonality of a factor this population for effective analysis, so as to develop a targeted feedback mechanism design strategy.

The Impact of User Behavior. Interactive design can be understood in nature as a behavioral design. In the product interaction design, the user in a certain scene under the specific operational behavior of course should be the designer's attention. Specific user behavior can be varied, but to reveal its essence, they have a certain degree of commonality. In other words, the designer can 
characterize the user behavior as a certain behavior.

The Impact of Users Use Scene. Users use the product where the scene at a great level affects the product feedback mechanism type, or even play a decisive role. For example, users can use the scene is divided into three kinds of representative occasions: noisy downtown, warm family, business-oriented companies. Noisy city center must pay attention to F1, F2 design, because the operation of the environment by the impact, it is often reflected in the public products; and warm family is very noticeable to the design of F3, the quality of the family is very important pursuit Target; business-oriented companies will have to pay attention to the design of F2, because the company is a very time about the concept of the occasion.

\section{Conclusion}

Interactive design research on feedback mechanisms has just started, but designers have been increasingly concerned about the great prospects of feedback in the interactive products. Through the user-centered design research, the proportion of feedback mechanism in a successful product is more and more. The feedback analysis should carefully consider the user's awareness and acceptance and make the feedback mechanism and comprehensive human behavior into a situation and get a harmonious and unified human-computer interaction system.

\section{Acknowledgements}

Fund Project: Lanzhou Jiaotong University Youth Fund (2016031)

\section{References}

[1] Liu Kang, Jiang Xiao, Li Shi-guo. Application of Feedback Mechanism in Product Interaction Design[J].Packaging Engineering, 2009, 30(11): 123-125

[2] Tong Qing, Jiang Xiao. Research of Interaction Architecture of Mobile Application Software[J].Packaging Engineering, 2011, 32(6): 67-70

[3] Wang Yue-feng, Jiang Xiao. Research on Feedback Mechanism in the Internet Product Design[J].Packaging Engineering, 2011, 32(12):97-100.

[4] Cheng Bin, Zhao Hong-mei. Discussion on Matching Principle Applied in Human-computer Interaction Design[J].Packaging Engineering,2008,29(11):125-127

[5] Norman D A. The Design of Future Things[M].Beijing: Publishing House of Electronics Industry, 2009 